\title{
A Comparison between Laparoendoscopic Single-Site and Conventional Laparoscopic Renal Stone Removal Surgery: A Study of Application in Two Cases
}

\author{
Xingqiao Wen", Yi Cai, Yiran Tao, Baoyi Zhu, Chunwei Ye, Cheng Hu, Yu Wang \\ Department of Urology, The Third Affiliated Hospital of Sun Yat-sen University, Guangzhou, China \\ Email: *xingqiaowen@yahoo.com
}

Received May 21, 2012; revised June 25, 2012; accepted July 3, 2012

\begin{abstract}
Aim: To present our initial experience of applying laparoendoscopic single-site surgery (LESS) nephrolithotomy in the treatment of renal calculi. Methods: In August 2010 and April 2012, the LESS nephrolithotomy technique was applied to treat two patients with a renal calculus. One patient had a calculus measuring $5 \times 3 \mathrm{~cm}$, and the second patient's measured $3 \times 3 \mathrm{~cm}$. Both patients underwent general anesthesia. The operations were performed through a retroperitoneal approach with a single-port instrument inserted through the retroperitoneal incision. The standard laparoscopic retroperitoneal technique was performed, and the calculi were removed by incising the area of the thin renal cortex and the renal pelvis, respectively. The operating time, estimated blood loss, intraoperative complications, other complications, drainage time, and post-operative hospital stay were recorded. Results: Both procedures were completed successfully. No additional trocars were added. No conversion to standard laparoscopic or open surgery was needed. The operating times were $130 \mathrm{~min}$ and $120 \mathrm{~min}$, and the estimated blood losses were $40 \mathrm{~mL}$ and $30 \mathrm{~mL}$. There were no intraoperative complications. The wound drainage times were $3 \mathrm{~d}$ and $2 \mathrm{~d}$, respectively and the post-operative hospital stays were $8 \mathrm{~d}$ and $7 \mathrm{~d}$, respectively. There was no secondary bleeding or wound infection. Follow-up times were 2 years and 3 months, respectively. No incidence of hydronephrosis was recorded. Conclusions: LESS nephrolithotomy is safe and feasible for treating kidney calculi. It can be applied in patients with large renal calculi and regionally thin renal parenchyma.
\end{abstract}

Keywords: Laparoendoscopic Single-Site Surgery; Renal Calculi; Nephrolithotomy

\section{Introduction}

In recent years, laparoscopic nephrolithotomy had been applied to treat single large renal calculi with a thin, weak renal cortex $[1,2]$. Laparoendoscopic single-site surgery (LESS) is a new approach that has been implemented in the clinical setting in the past few years. During the LESS approach, all instruments are inserted through a natural orifice or a single incision in the body. This approach results in a decreased number of scars and an improved cosmetic effect, which is desired by certain patients $[3,4]$.

The clinical application of laparoendoscopic singlesite surgery in treating renal calculi has been rarely reported. Recently, we used this approach to treat two patients. Our initial experiences are presented here.

\section{Material and Method}

\subsection{Clinical Data}

Case 1: Female, 37 years old, pain in the left waist for 10

${ }^{*}$ Corresponding author. months. CT scan found a left renal calculus, $5 \times 3 \mathrm{~cm}$, with dilated regional kidney calices, a thin cortex and mild renal hydronephrosis (Figure 1).

Left renal calculi, $5 \times 3 \mathrm{~cm}$. regional kidney calices dilated, with thin cortex and mild renal hydronephrosis. The right kidney calculi had been removed in the previous surgery.

Case 2: Male, 36 years old, pain in the left waist for three months. Left renal pelvic calculus, $3 \times 3 \mathrm{~cm}$, with a dilated renal pelvis (Figure 2).

\subsection{Surgical Technique}

Case 1: The patient was placed under general anesthesia. Retroperitoneal approach was used. A self-made balloon was used to establish the retroperitoneal operative cavity. Triport (Olympus) was inserted through a vertical $2.5 \mathrm{~cm}$ skin incision placed above the crest of the iliac spine in the left mid-axillary line. The Triport is a multichannel access port that allows the simultaneous passage of up to three laparoscopic instruments through the same incision. 

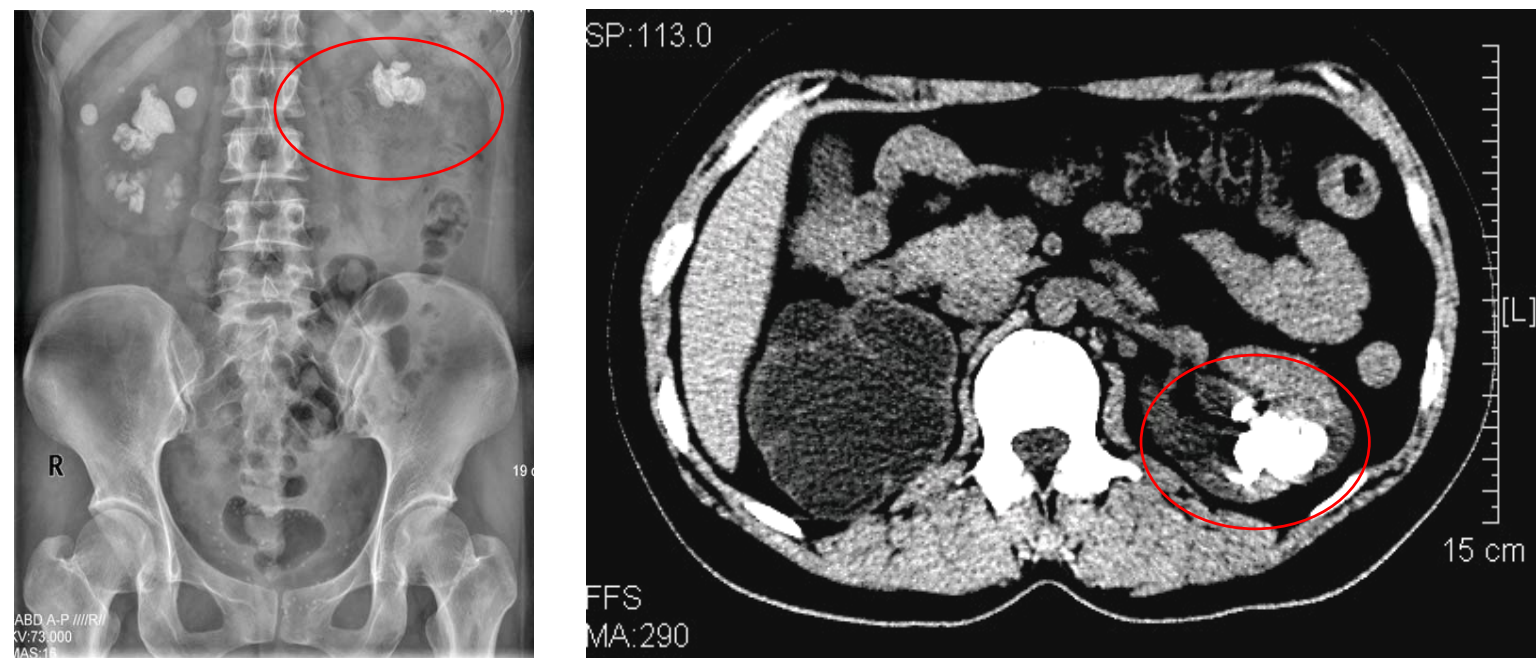

Figure 1. The preoperative images of case 1 (KUB and CT).
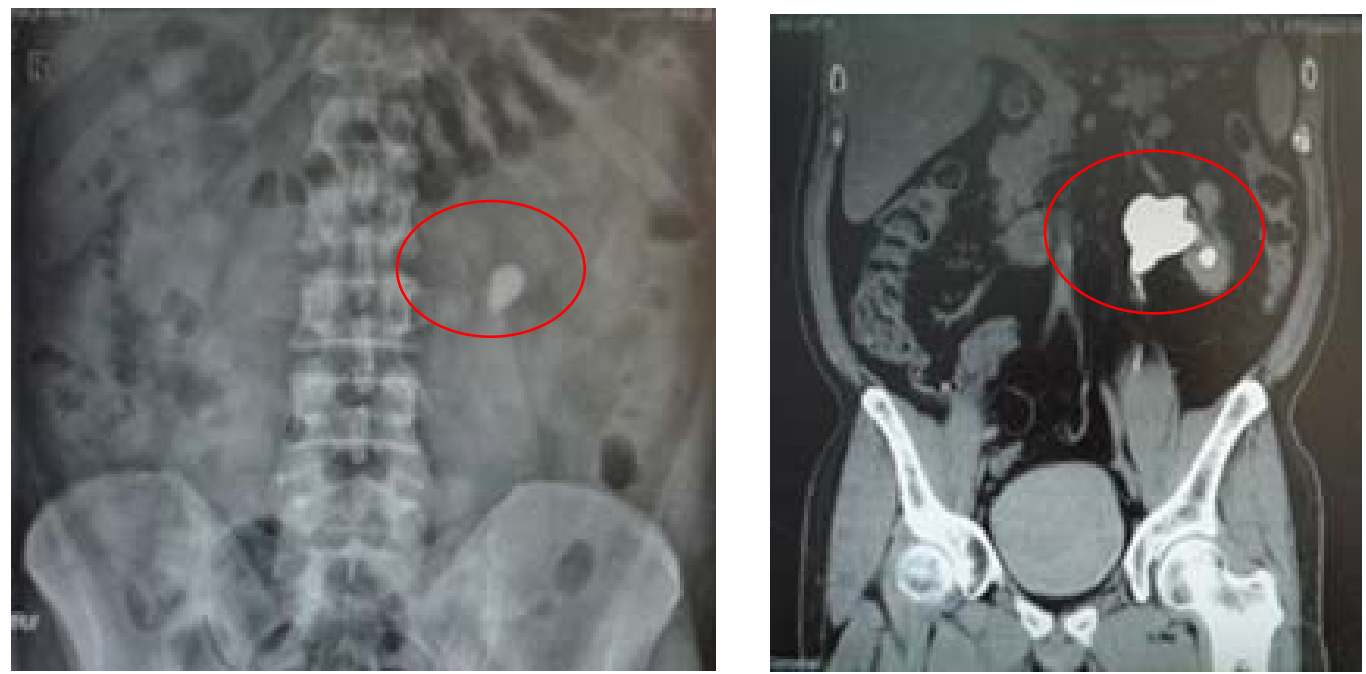

Figure 2. The preoperative images of case 2 (KUB and CT). The left renal pelvic calculus, the size was $3 \times 3 \mathrm{~cm}$. With dilated renal pelvic.

After insufflation of the retroperitoneal cavity with $\mathrm{CO}_{2}$ to a pressure of $13 \mathrm{mmHg}$, a $5 \mathrm{~mm}$ flexible tip laparoscope with an integrated camera head was inserted. An operative triangulation was created using a grasper and shear, an electrocautery hook, and a $5 \mathrm{~mm}$ laparoscopic harmonic scalpel.

After dissecting the perirenal fat, a weak region of the kidney was found and the renal calculus was palpated. After the renal pedicle was isolated, the renal artery was completely dissected and then was clamped temporarily by a bulldog clamp. The renal parenchyma was incised above the stone with the electrocautery hook and the harmonic scalpel. The calculus was taken out intact and then removed from the body in a sterile bag. A 3-0 absorbable Vicryl was used to close the renal incision. The bulldog clamp was removed from the renal artery. An external retroperitoneal drain was placed in the retro- peritoneal cavity at the completion of the procedure, which was removed after 3 days if no urinary leakage was present (Figure 3).

Case 2: The operative cavity was established using the method described in Case 1. A Triport was inserted. The ureter was identified along the psoas major muscle. Adipose tissue was separated to expose the renal pelvis. Without blocking the renal artery, the mucosa of the renal pelvis was sliced above the calculus with an electroknife, and the stone was drawn out intact (Figure 4). With the guidance of a flexible guide wire, a double $\mathrm{J}$ stent $(4.7 \mathrm{~F})$ was inserted into the ureter for drainage and proper placement was confirmed. An interrupted suture using 3-0 Vicryl was applied to close the renal pelvis incision. An external retroperitoneal drain was placed in the retroperitoneal cavity after the operation, which was removed after 3 days if no urinary leakage was present. 

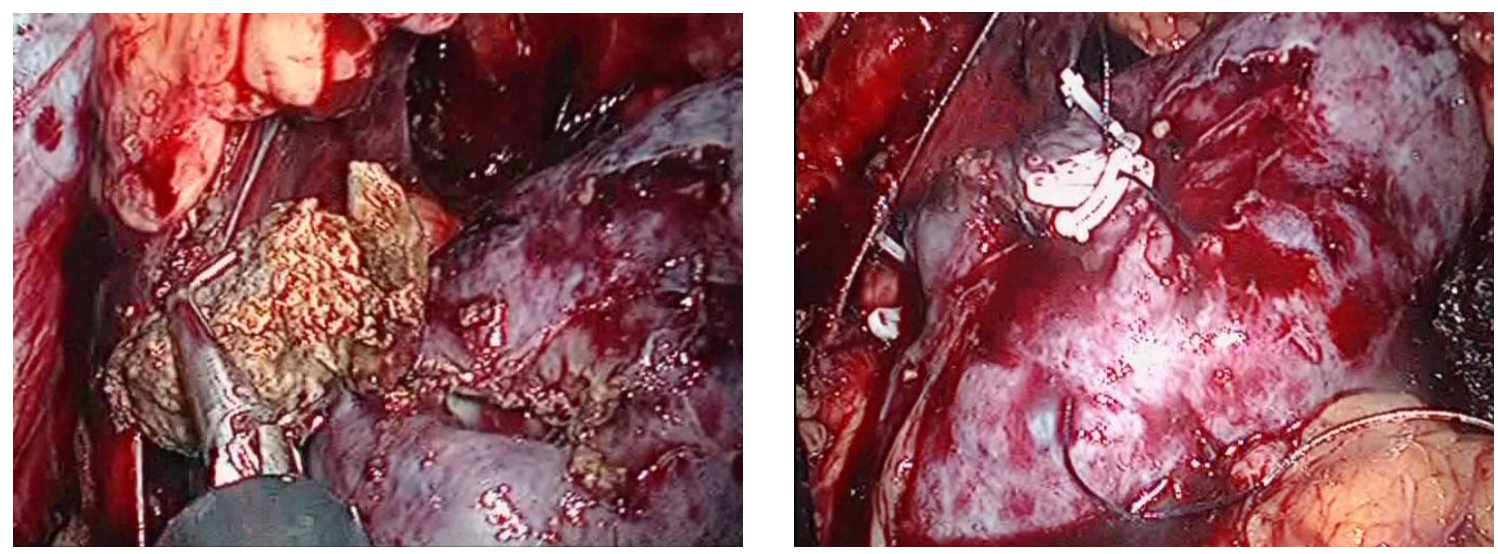

Figure 3. Intraoperative photos of case 1. The left kidney stone was taken out. The incision of the renal parenchyma was sutured completely.
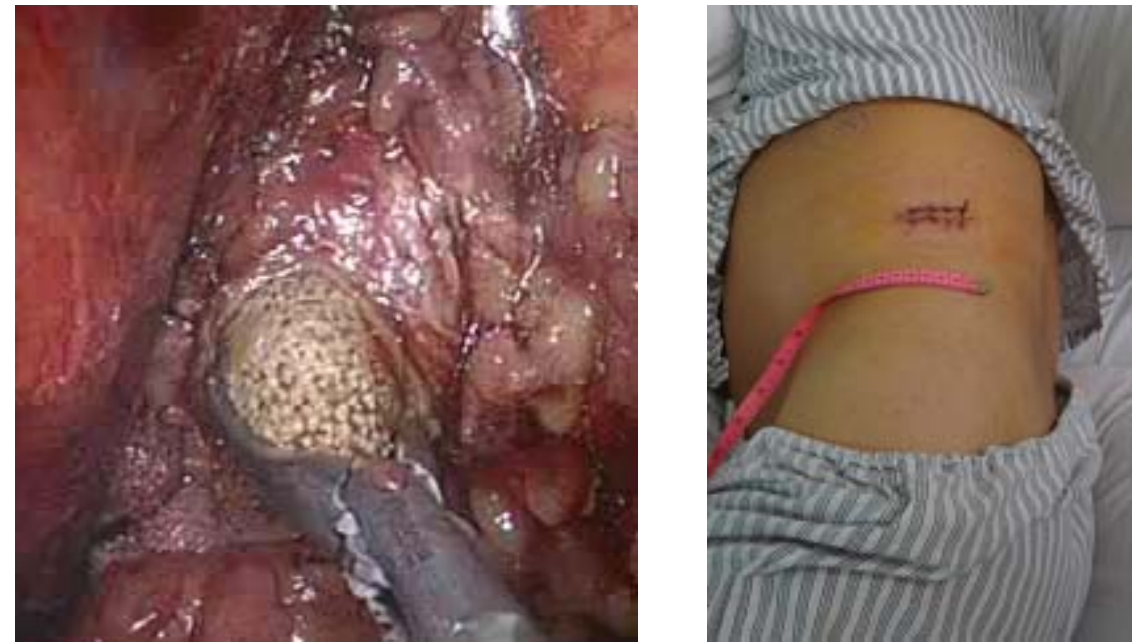

Figure 4. Intraoperative photos of case 2. Remove the stone from the renal pelvis. Only a small incision was seen on the skin after the surgery.

\subsection{Monitoring Index}

The operating time, estimated blood loss, intraoperative complications, post-operative hospital stay, and retroperitoneal drainage time were recorded for both cases.

\subsection{Follow-Up}

B-ultrasound of the urinary system and an abdominal $\mathrm{X}$-ray were performed 1 month after surgery to evaluate for retroperitoneal hematoma or urine leakage and to confirm the position of the double J stents.

\section{Results}

Both operations were completed successfully. Neither case required conversion to traditional laparoscopic or open surgery. All calculi were cleared completely.

Neither patient experienced urine leakage. No other complications such as adjacent organ (bowel and blood vessels) damage or urinary tract infection were recorded.
Case 1 had a blood loss of $40 \mathrm{~mL}$ and case 2 had a blood loss of $30 \mathrm{~mL}$. Postoperative days in hospital were $8 \mathrm{~d}$ and $7 \mathrm{~d}$, respectively.

Patients were followed for 2 years and 3 months, respectively. Both patients achieved a cure without renal pelvis narrowing or hydronephrosis.

\section{Discussion}

Traditionally, laparoscopic surgery requires three to five skin incisions, which result in several scars. LESS surgery requires only one small incision, and it has been applied successfully in a variety of urological surgeries $[3,4]$.

In recent years, percutaneous nephrolithotomy (PCNL) has been widely used in treating kidney stones and has achieved good efficacy. Laparoscopic nephrolithotomy has unique advantages, including the ability to cut the renal cortex directly, thereby removing the calculus completely. Laparoendoscopic single-site surgery nephrolitho- 
tomy has not been widely used. The initial experience with the two patients in this study demonstrates that this procedure is feasible and efficient. It has several advantages, such as fewer incisions, less invasiveness, and a better cosmetic effect. It has been welcomed by patients who desire an improved cosmetic outcome.

However, in the clinical application of this procedure, choosing the appropriate indications is very important. The patients should have regionally weak and easy-toincise renal parenchyma. To treat patients with a thick renal parenchyma, the renal artery would need to be temporarily blocked. Suturing the incisions of the renal parenchyma or the renal pelvis properly is another important step in preventing urine leakage and blood loss.

Compared with the traditional laparoscopic surgery, LESS surgery has a unique learning curve, principally because of the difficulty in navigating the instruments with a limited range of motion and the need for significant coordination between the surgeon and the camera holder $[5,6]$. Because all of the instruments are in close proximity, clashing of the instruments and the laparoscope is common $[7,8]$. During the operation, placing several parallel instruments makes triangulation more difficult. Another limitation is the lack of additional assistant trocars and effective retraction, which makes it difficult to properly insert the ureteral stents.

The application of flexible laparoscopic single-site operation equipment can reduce the crowding of equipment. In addition, a flexible endoscope assists in exposing a wider operative field. The application of some adaptive operating skills can reduce the surgical difficulties and shorten the operating time. For example, after suturing, the two ends of the suture can be clamped with the absorbable Lapro-clip (Tyco Company), which can substitute for the knot-tying process [9]. Additionally, placing a microcatheter stent in a double $\mathrm{J}$ tube (made by the stent of a pediatric urethral catheter) can help to make the ends of double $\mathrm{J}$ tube easier to insert [10,11]. A single-site operation device, such as a TriPort, or one made using routine surgical gloves and two rings, can be employed. The latter device is simple, easily made, and low cost but is also easily broken [11].

With the development of various single port devices, flexible laparoscopes, and other instruments, the difficulties with LESS nephrolithotomy can be reduced.

\section{Conclusion}

In summary, LESS nephrolithotomy and pyelolithotomy are feasible and effective for treating kidney stones. This procedure can be applied in patients with large renal calculi and regionally thin renal parenchyma. The results of long-term follow-up and the application in a larger population are warranted to further characterize the benefits of this technique.

\section{REFERENCES}

[1] A. Hoznek, J. Rode, I. Ouzaid, et al., "Modified Supine Percutaneous Nephrolithotomy for Large Kidney and Ureteral Stones: Technique and Results," European Urology, Vol. 61, No. 1, 2012, pp. 164-170. doi:10.1016/j.eururo.2011.04.031

[2] C. Giedelman, J. Arriaga, O. Carmona, et al., "Laparoscopic Anatrophic Nephrolithotomy: Developments of the Technique in the Era of Minimally Invasive Surgery," Journal of Endourology, Vol. 26, No. 5, 2012, pp. 444450. doi:10.1089/end.2011.0193

[3] R. Autorino, J. A. Cadeddu, M. M. Desai, et al., "Laparoendoscopic Single-Site and Natural Orifice Transluminal Endoscopic Surgery in Urology: A Critical Analysis of the Literature," European Urology, Vol. 59, No. 1, 2011, pp. 26-45. doi:10.1016/j.eururo.2010.08.030

[4] J. U. Stolzenburg, P. Kallidonis, H. Till, et al., "Current Status of Laparoendoscopic Single-Site Surgery in Urology," World Journal of Urology, Vol. 27, No. 6, 2009, pp. 767-773. doi:10.1007/s00345-009-0459-0

[5] W. M. White, G. P. Haber, R. K. Goel, et al., "SinglePort Urological Surgery: Single-Center Experience with the First 100 Cases," Urology, Vol. 74, No. 4, 2009, pp. 801-804. doi:10.1016/j.urology.2009.04.030

[6] M. M. Desai, A. K. Berger, R. Brandina, et al., "Laparoendoscopic Single-Site Surgery: Initial Hundred Patients," Urology, Vol. 74, No. 4, 2009, pp. 805-812. doi:10.1016/j.urology.2009.02.083

[7] A. Rane and P. Rao, "Single-Port-Access Nephrectomy and Other Laparoscopic Urologic Procedures Using a Novel Laparoscopic Port (R-Port)," Urology, Vol. 72, No. 2, 2008, pp. 260-263. doi:10.1016/j.urology.2008.01.078

[8] X. Q. Wen, W. T. Huang, J. M. Zheng, et al., "Experience of Building a Pig Model for the Training of Laparoendoscopic Single-Site Surgery," Chinese Journal Endourology, Vol. 5, No. 1, 2011, pp. 14-17.

[9] X. Q. Wen, W. T. Huang, J. Situ, et al., "Single-Port Laparoscopic Radical Prostatectomy: Initial Experience and Technical Points to Reduce Its Difficulties," Chinese Medical Journal, Vol. 124, No. 23, 2011, pp. 4092-4095.

[10] X. Wen, X. Li, J. Situ, et al., “Application of a Temporary Ureter Clamp for Retroperitoneal Laparoscopic Ureterolithotomy," World Journal of Urology, Vol. 28, No. 1, 2010, pp. 99-102. doi:10.1007/s00345-009-0424-y

[11] X. Wen, X. Liu, H. Huang, et al., "Retroperitoneal Laparoendoscopic Single-Site Ureterolithotomy: A Comparison with Conventional Laparoscopic Surgery," Journal of Endourology, Vol. 26, No. 4, 2012, pp. 366-371. doi:10.1089/end.2011.0330 